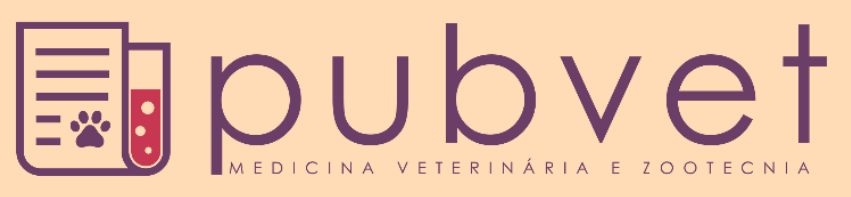

https://doi.org/10.31533/pubvet.v12n12a238.1-5

\title{
Gato x gestante. Avaliação do conhecimento da população sobre a toxoplasmose
}

\author{
Ed Wilson Santos ${ }^{1 *}$, Silvio L. Souza ${ }^{1} \bullet$, Cesar Augusto Dinola Pereira $^{1 \bullet}$ \\ ${ }^{\text {I} P r o f e s s o r ~ d a ~ F a c u l d a d e ~ d e ~ M e d i c i n a ~ V e t e r i n a ́ r i a ~ d a ~ U n i v e r s i d a d e ~ A n h e m b i ~ M o r u m b i, ~ S a ̃ o ~ P a u l o, ~ S P / B r a s i l . ~ E-m a i l: ~ * A u t o r ~}$ \\ para correspondência, E-mail: ed.wilson@usp.br
}

RESUMO. A Toxoplasmose é uma zoonose causada pelo protozoário Toxoplasma gondii que pode ser transmitida ao homem principalmente pela ingestão de alimentos contaminados, como frutas e verduras, além de carne crua ou mau cozida contendo cistos. Apesar dos felinos terem um papel muito importante no ciclo da doença, desenvolvendo a reprodução sexuada do protozoário, o contato com o gato envolve parte muito baixa na transmissão da doença. Esse estudo avaliou o conhecimento da população em relação ao papel epidemiológico do gato. Foi observado que muitos desconhecem a principal forma de transmissão da toxoplasmose e culpam o gato, sugerindo, inclusive, o distanciamento da gestante durante a gestação.

Palavras chave: gato, gestação, toxoplasmose, transmissão

\section{Cat $x$ pregnant. Assessment of population knowledge about toxoplasmosis}

\begin{abstract}
Toxoplasmosis is a zoonosis caused by the protozoan Toxoplasma gondii that can be transmitted to humans mainly by eating contaminated foods such as fruits and vegetables, as well as raw or undercooked meat containing cysts. Although cats have a very important role in the disease cycle, by developing the sexual reproduction of the protozoan, contact with the cat involves a very low part in the transmission of the disease. This study evaluated the knowledge of the population regarding the epidemiological role of the cat. It was observed that many are unaware of the main form of transmission of toxoplasmosis and blame the cat, even suggesting the distancing of the pregnant woman during pregnancyo.
\end{abstract}

Keywords: cat, gestation, toxoplasmosis, transmission

\section{Gato $x$ gestante. Evaluación del conocimiento de la población sobre la toxoplasmosis}

RESUMEN. La Toxoplasmosis es una zoonosis causada por el protozoario Toxoplasma gondii que puede ser transmitida al hombre principalmente por la ingestión de alimentos contaminados, como frutas y verduras, además de carne cruda o mal cocida conteniendo quistes. Aunque los felinos desempeñan un papel muy importante en el ciclo de la enfermedad, desarrollando la reproducción sexuada del protozoario, el contacto con el gato involucra parte muy baja en la transmisión de la enfermedad. Este estudio evaluó el conocimiento de la población en relación al papel epidemiológico del gato. Se observó que muchos desconocen la principal forma de transmisión de la toxoplasmosis y culpan al gato, sugiriendo, inclusive, el distanciamiento de la gestante durante la gestación.

Palabras clave: gato, gestación, toxoplasmosis, transmisión 


\section{Introdução}

A Toxoplasmose é uma zoonose com incidência mundial, causada por um protozoário Toxoplasma gondii (Silva et al. 2006). Dentro da sua epidemiologia, os felinos domésticos e silvestres são considerados os únicos hospedeiros definitivos, pois estes eliminam os oocistos pelas fezes, levando à contaminação do meio-ambiente. $\mathrm{O}$ homem e os animais homeotérmicos são tidos como hospedeiros intermediários, pois são neles que o parasita efetua seu ciclo extra-intestinal, não chegando à sua fase adulta (Negri et al. 2008; Amendoeira \& Coura 2010).

A toxoplasmose é considerada uma das infecções mais difundidas do mundo, tendo soro positividade de $10 \%$ a $90 \%$ dependendo da região. Sua principal forma de transmissão é por ingestão de alimentos contaminados, como frutas e verduras, além de carne crua ou mau cozida contendo cistos. O felino, como hospedeiro definitivo, promove a liberação de oocistos nas fezes, o que pode levar a infeção de outros hospedeiros por contato com as fezes (Torgerson \& Mastroiacovo 2013).

Em humanos, a maioria dos casos é assintomática, vindo a manifestar-se em indivíduos suprimidos através de alterações neurológicas e processos inflamatórios em diversos tecidos (Hill et al. 2005; MitsukaBreganó et al. 2010). Possui grande relevância por causar doenças congênitas em humanos e em diversas outras espécies de hospedeiros intermediários, além de abortamentos (Silva et al. 2006).

A prevenção consiste em estratégias higiênicosanitário, evitando a ingestão de vegetais mal higienizados e produtos de origem animal crus ou mal cozidos. Ainda, utilizar luvas para atividades que precisem contato com areia como jardinagem e para limpar as caixas de areia dos gatos (Prado et al. 2011).
Sabendo-se que a transmissão pode ocorrer tanto por alimentos contaminados como por contato com as fezes do gato, nosso objetivo nesse trabalho foi avaliar o conhecimento da população a respeito do ciclo da doença, sua relação com a gestante e a melhor forma de prevenção.

\section{Material e métodos}

Foram realizados 420 questionários por alunos do $9^{\circ}$ semestre do curso de Medicina Veterinária da Universidade Anhembi Morumbi. Esses questionários foram realizados em diversos locais para se obter uma maior abrangência sobre o conhecimento da transmissão da toxoplasmose e o perigo para gestantes. Foram abordadas 196 gestantes usuários do Sistema Único de Saúde; 70 usuários do metrô que acessaram pela estação Bresser-Mooca; 64 usuários do pet shop Petz localizado na Rua Augusta, São Paulo; 40 Alunos de medicina da Universidade Anhembi Morumbi de todos os semestres; 40 usuários do Hospital Veterinário da Universidade Anhembi Morumbi; 40 professores do ensino fundamental e médio do sistema público de ensino.

As perguntas realizadas foram: "Qual a principal forma de transmissão da toxoplasmose?"; "É seguro ter contato com gatos durante a gestação?"; "Quais as principais formas de prevenção para a toxoplasmose."

\section{Resultados}

Em nossa avaliação, a respeito do conhecimento da principal forma de transmissão da toxoplasmose (Tabela 1), observamos que estudantes de medicina e usuários de pet shops tem um melhor conhecimento a esse respeito, sendo que mais de $80 \%$ apontaram os alimentos como principal fator. Entre os professores de ensino fundamental e usuários do hospital veterinário, os alimentos tiveram uma porcentagem de respostas muito próxima ao contato com gatos.

Tabela 1. Qual a principal forma de transmissão da toxoplasmose?

\begin{tabular}{lcccccc}
\hline & \multicolumn{2}{c}{ Contato com o gato } & \multicolumn{2}{c}{ Alimentos contaminados } & \multicolumn{2}{c}{ Outros } \\
\hline Público avaliado & F.R. & F.A. & F.R. & F.A. & F.R. & F.A. \\
\hline Estudantes de medicina & $17,50 \%$ & 7 & $82,50 \%$ & 33 & $0 \%$ & 0 \\
Professores do ensino fundamental & $37,50 \%$ & 15 & $40 \%$ & 16 & $22,50 \%$ & 9 \\
Usuários do Hospital Veterinário & $42 \%$ & 18 & $46 \%$ & 19 & $12 \%$ & 3 \\
Usuários de pet shops & $14,30 \%$ & 9 & $82,60 \%$ & 53 & 3,1 & 2 \\
Usuários do metrô & $20 \%$ & 14 & $68,60 \%$ & 48 & $11,40 \%$ & 8 \\
Gestantes & $60,70 \%$ & 119 & $31,60 \%$ & 62 & $7,70 \%$ & 15 \\
\hline
\end{tabular}

F.R. - Frequência Relativa; F.A. - Frequência absoluta. 
Os usuários do metrô compõem um grupo bem heterogêneo, com pessoas de diversas áreas. Nesse grupo observamos que $68,6 \%$ acertaram a questão. Assim como em outros grupos, ocorreram várias respostas equivocadas, como a transmissão por mosquitos, contato direto com doentes e mordidas de cães.

Apesar da maioria dos grupos pesquisados terem apontado os alimentos como principal forma de transmissão da toxoplasmose, observamos que o grupo de gestantes apresenta a maior porcentagem $(60,7 \%)$ que acredita que o gato é a principal causa.

A segunda pergunta da pesquisa era sobre o contato com os gatos durante a gestação (Tabela 2). A maioria dos grupos acredita que não há problemas, sendo o grupo de usuários do pet shop $(86 \%)$ os mais seguros em relação ao contato. Os usuários do metrô $(78,5 \%)$, usuários do hospital veterinário $(70 \%)$, professores de ensino fundamental $(70 \%)$ e estudantes de medicina (65\%) também tiveram uma elevada porcentagem de acerto. Mais uma vez, o grupo de gestantes mostrou grande preocupação com o contato com os gatos, sendo que apenas $28 \%$ delas acreditam que o contato com gatos na gestação não é prejudicial.
Em relação à melhor forma de prevenção (Gráfico 1) foi dado um quadro com diversos métodos. Alguns errados e outros certos. As pessoas poderiam assinalar mais de uma questão se considerassem como correta. A maioria das pessoas acertou quais as principais formas de prevenção: não comer alimentos mal lavados e carnes cruas $(91 \%)$ e lavar as mãos após contato com gatos e seus utensílios ( $83 \%$ ). Outra forma de prevenção importante que foi apontada por uma menos quantidade de pessoas é a lavagem das mãos após o preparo de alimentos (35\%). No entanto, há muitas pessoas que colocaram alternativas como vacinação (20\%), vermifugação (43\%), manter os gatos distantes (15\%) e até mesmo evitar água parada $(2,6 \%)$.

\section{Discussão}

A toxoplasmose é uma zoonose que causará problemas em pessoas com doenças imunossupressoras ou em momento de gestação, levando a alterações no desenvolvimento do feto ou mesmo abortamento. Por isso, sua forma de transmissão deve ser muito bem compreendida e ensinada, evitando mitos e outros prejuízos à saúde pública e animal (Varella et al. 2003).

Tabela 2. É seguro ter contato com gatos durante a gestação?

\begin{tabular}{|c|c|c|c|c|}
\hline \multirow[b]{2}{*}{ Público avaliado } & \multicolumn{2}{|c|}{ Sim } & \multicolumn{2}{|c|}{ Não } \\
\hline & F.R. & F.A. & F.R. & F.A. \\
\hline Estudantes de medicina & $65 \%$ & 26 & $35 \%$ & 14 \\
\hline Professores do ensino fundamental & $70 \%$ & 28 & $30 \%$ & 12 \\
\hline Usuários do Hospital Veterinário & $70 \%$ & 28 & $30 \%$ & 12 \\
\hline Usuários de pet shops & $86 \%$ & 55 & $14 \%$ & 9 \\
\hline Usuários do metrô & $78,50 \%$ & 55 & $21,50 \%$ & 15 \\
\hline Gestantes & $27,60 \%$ & 54 & $72,40 \%$ & 142 \\
\hline
\end{tabular}

F.R. - Frequência Relativa; F.A. - Frequência absoluta.

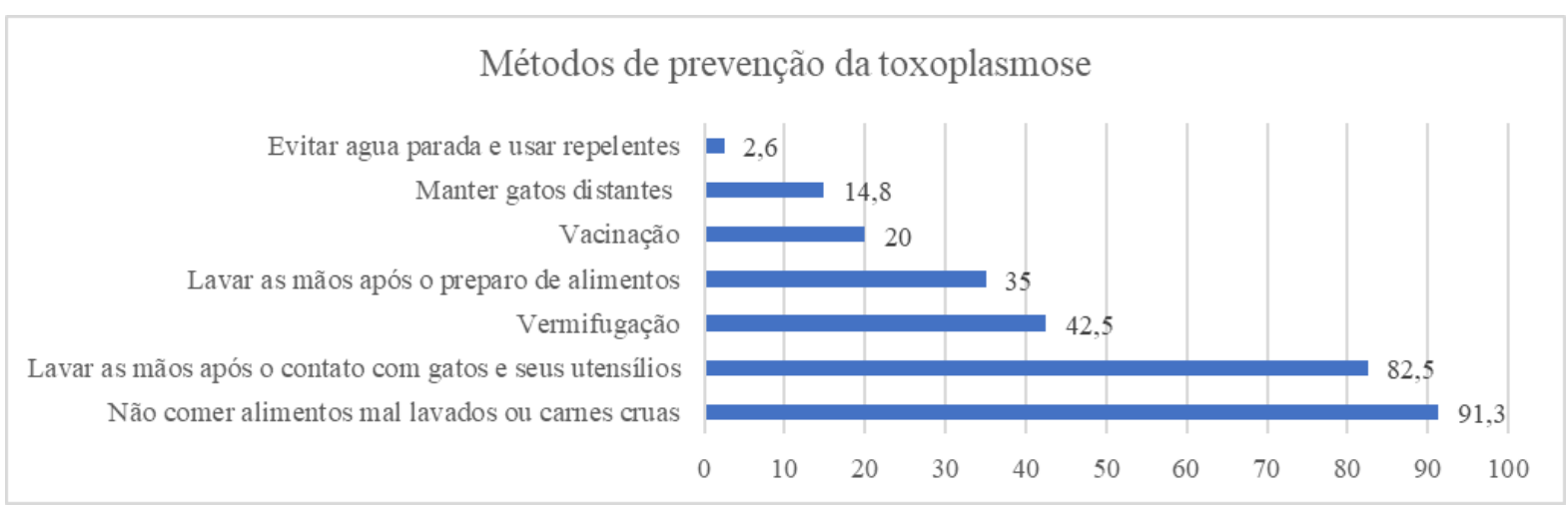

Gráfico 1. Quais as principais formas de prevenção para a toxoplasmose (pode assinalar mais de uma questão) 
É importante ressaltar que não se trata de uma doença de países subdesenvolvidos, pois é encontrada em grande prevalência em cidades de países desenvolvidos, como Paris (65\%), Nova Iorque (32\%) (Remington et al. 2001) e Londres (19\%) (Gilbert et al. 1993). Em cidades brasileiras, a porcentagem também é bem variável, ficando por volta de $77 \%$ no Rio de Janeiro (Meirelles Filho 1985) e 32\% em São Paulo (Gilbert et al. 1993).

Os gatos, como hospedeiros definitivos, apresentam o oocisto nas fezes. No entanto, poderão transmitir a toxoplasmose apenas durante 7 a 10 dias de sua vida. Desde o momento que foram infectados, até o momento de seu organismo ter produzido os anticorpos e eliminar o agente, deixando anticorpos $\operatorname{IgG}$ que servirão como memória imunológica. Apesar desse curto período de transmissão e de estudos demonstrarem que o contato com gato representa baixa incidência de transmissão, ainda há muitas pessoas que associam essa como principal forma de contágio, principalmente em grupos mais envolvidos, como o caso das gestantes que, naturalmente, apresentam medo de alterações no feto.

$\mathrm{O}$ receio das gestantes fica claro ao perguntarmos se o contato com gatos é seguro à gestante. Nos diversos grupos pesquisados constatamos que 65 a $86 \%$ das pessoas consideram essa relação segura, mas no grupo de gestantes esse número cai para 27,6\%. Ao avaliarmos o melhor método de prevenção, foi observado que mais de $82 \%$ das pessoas tem conhecimento que a higiene pessoal é fundamental após o contato com os gatos e seus utensílios, pois podem, eventualmente, estar contaminados pelos oocistos. Sem dúvida, em conjunto com a anterior, a melhor forma profilática seria uma boa higiene alimentar, promovendo a correta lavagem e preparação de vegetais, bem como o cozimento adequado da carne, em temperaturas acima de $66^{\circ}$ C. Não devemos esquecer também de beber e utilizar para o preparo das refeições a água tratada, além de lavar as mãos antes das refeições e após o manuseio de carne crua (Silva et al. 2006).

A vermifugação também foi lembrada por 43\%. No entanto, há muitas respostas equivocadas. A vacinação foi apontada por $20 \%$ dos pesquisados como sendo uma forma eficaz, no entanto, não há vacinas contra toxoplasmose. Há, ainda, $15 \%$ que insistem em manter distância dos gatos, mesmo essa sendo uma forma rara de transmissão. Outras respostas, como evitar agua parada e uso de repelentes foram citadas por pessoas que demonstram não saber o que é toxoplasmose e não ter conhecimento de seu ciclo epidemiológico.

\section{Conclusão}

A toxoplasmose é uma zoonose transmitida, principalmente, por alimentos contaminados. Apesar do contato com o gato ser uma pequena parcela do contágio, há ainda muitas pessoas que não consideram seguro o contato com a gestante. É fundamental uma melhor educação da população para conscientização e entendimento que uma boa higiene alimentar e pessoal são, sem dúvidas, os melhores métodos profiláticos contra a toxoplasmose.

\section{Agradecimentos}

Alunos do $9^{\circ}$ Semestre do curso de Medicina Veterinária da Universidade Anhembi Morumbi em 2018, pela realização dos questionários.

\section{Referências bibliográficas}

Amendoeira M.R.R. \& Coura L.F.C. 2010. Uma breve revisão sobre toxoplasmose na gestação. Scientia Medica 20, 113-119.

Gilbert R.E., Tookey P.A., Cubitt W.D., Ades A.E., Masters J. \& Peckham C.S. 1993. Prevalence of toxoplasma IgG among pregnant women in west London according to country of birth and ethnic group. BMJ: British Medical Journal 306, 185.

Hill D.E., Chirukandoth S. \& Dubey J.P. 2005. Biology and epidemiology of Toxoplasma gondii in man and animals. Animal Health Research Reviews 6, 41-61.

Meirelles Filho J. 1985. Toxoplasmose e gravidez: inquérito sorológico em gestantes e seus recém-nascidos na Maternidade-Escola da Universidade Federal do Rio de Janeiro. Jornal Brasileiro de Ginecologia 95, 393-401.

Mitsuka-Breganó R., Lopes-Mori F.M.R. \& Navarro I.T. 2010. Toxoplasmose adquirida na gestação e congênita: vigilância em saúde, diagnóstico, tratamento e condutas. Eduel, Londrina, Paraná, Brasil.

Negri D., Cirilo M.B., Salvarane R.S. \& Neves M.F. 2008. Toxoplasmose em cães e gatos. Revista Científica de Medicina Veterinária. Garça, SP: FAEF 1, 1-7. 
Prado A.A.F., Almeida G.F., Gontijo L.S. \& Torres M.L.M. 2011. Toxoplasmose: o que o profissional da saúde deve saber. Enciclopédia Biosfera 7, 1-30.

Remington J.S., McLeod R., Thulliez P. \& Desmonts G. 2001. Toxoplasmosis. In: Infectious diseases of the fetus and newborn infant (eds. by Remington JS \& Klein JO), pp. 205-346. WB Saunders, Philadelphia, USA.

Silva F.W.S., N.D. A., Amóra S.S.A., F.H.V. T., Accioly M.P., Carvalho C.G., Nóbrega R.M., Filgueira K.D. \& Feijó F.M.C. 2006. Toxoplasmose: uma revisão. Ciência Animal 16, 71-77.
Torgerson P.R. \& Mastroiacovo P. 2013. The global burden of congenital toxoplasmosis: a systematic review. Bulletin of the World Health Organization 91, 501-508.

Varella I.R.d.S., Wagner M.B., Darela A.C., Nunes L.M. \& Müller R.W. 2003. Prevalência de soropositividade para toxoplasmose em gestantes. Jornal de Pediatria 79, 69-74.

Recebido: 9 novembro, 2018.

Aprovado: 30 novembro, 2018.

Publicado: 27 dezembro, 2018.

Licenciamento: Este artigo é publicado na modalidade Acesso Aberto sob a licença Creative Commons Atribuição 4.0 (CC-BY 4.0),

a qual permite uso irrestrito, distribuição, reprodução em qualquer meio, desde que o autor e a fonte sejam devidamente creditados. 